\title{
Fraternal Twins with Cyclic Neutropenia: A Case Report of Full Dental Management
}

\author{
Mohammad A Alshoraim
}

\begin{abstract}
Aim: To report full dental treatment of 7-year-old fraternal twin girls diagnosed with cyclic neutropenia.

Background: Cyclic neutropenia is a rare autosomal-dominant disorder characterized by neutropenia that recurs every 14-35 days. It is estimated that the incidence of cyclic neutropenia is one to two per million. Clinically, it manifested by repetitive episodes of fever, susceptibility to infection, pharyngitis, recurrent mouth ulcerations, and periodontal diseases. Medical management includes supportive treatment, antibiotic therapy for infectious diseases, and granulocyte colony-stimulating factor (G-CSF), to raise blood neutrophils. Dental management of cyclic neutropenia patients varies from thorough oral hygiene, regular dental follow-ups, and surgical or nonsurgical periodontal treatment.

Case description: Seven-year-old fraternal twin girls diagnosed with cyclic neutropenia at the age of 6 months. They were referred from their pediatric hematologist after complaining of recurrent aphthous ulcer and extensive dental caries. Dental treatment started with preventive and supportive treatment and then full dental treatment under general anesthesia and maintenance visits for 6 months.

Conclusion: This report emphasizes the importance of dental treatment for patients with compromised medical conditions.

Clinical significance: The oral cavity is a potential source of inflammation or infection, and treating oral diseases could prevent serious systemic diseases.

Keywords: Cyclic neutropenia, Gingivitis, Oral cavity, Oral ulcers.

World Journal of Dentistry (2020): 10.5005/jp-journals-10015-1696
\end{abstract}

\section{INTRODUCTION}

Neutropenia is an abnormal decrease in the number of circulating neutrophils in the blood. It is described by the absolute neutrophil count (ANC). Human ANC differs in different population according to different factors. Generally, 1,500 cells/mm is used to describe neutropenia in most populations. ${ }^{1}$ Cyclic neutropenia is a rare autosomal-dominant disorder characterized by neutropenia that recurs every 14-35 days. Fluctuations in the neutrophils cells are due to oscillatory production of blood neutrophil counts by the bone marrow. ${ }^{2}$

Clinical manifestations vary in relation to the severity of neutropenia. Manifestations include repetitive episodes of fever, susceptibility to infection, pharyngitis, recurrent mouth ulcerations gingivitis, and gingival edema. Periodontitis and alveolar bone loss can result in premature tooth mobility and premature tooth loss in more severe cases. ${ }^{3}$ Medical management of cyclic neutropenia includes supportive treatment for fever and antibiotics for infectious diseases. Granulocyte colony-stimulating factor (G-CSF) is effective in the management of cyclic neutropenia. It is a glycoprotein that stimulates the bone marrow to produce granulocytes and stem cells into blood stream. ${ }^{4,5}$ Dental management of patient with cyclic neutropenia could be local or systemic management. Local management includes comprehensive oral hygiene, surgical or nonsurgical periodontal treatment, or extraction of severely affected teeth. Systemic administration of antibiotics and G-CSF are useful in treating the underlying cause and decreasing oral manifestations. ${ }^{4}$ This article reports full dental treatment of 7-year-old fraternal twin girls diagnosed with cyclic neutropenia with 6 months follow-up.

\section{Case Description}

Seven-year-old fraternal female twins born to nonconsanguineous Saudi parents came to the pediatric dental clinic in King Fahad
Department of Pediatric Dentistry, King Fahad Hospital, Jeddah, Kingdom of Saudi Arabia

Corresponding Author: Mohammad A Alshoraim, Department of Pediatric Dentistry, King Fahad Hospital, Jeddah, Kingdom of Saudi Arabia, Phone: +966 547944467, e-mail: maalshoraim@gmail.com

How to cite this article: Alshoraim MA. Fraternal Twins with Cyclic Neutropenia: A Case Report of Full Dental Management. World J Dent 2020;11(3):235-240.

Source of support: Nil

Conflict of interest: None

Hospital in Jeddah. They were referred from their pediatric hematologist after he noticed extensive dental caries and mouth ulceration in both girls. These twin girls were born prematurely after 31 gestation periods with low birth weight $(1.8 \mathrm{~kg}$ for the first patient and $1.6 \mathrm{~kg}$ for the twin). After birth, they suffered from respiratory distress syndrome and were admitted in the intensive care unit for care and monitoring for 3 weeks. At age 2 months, they visited the hospital because of recurrent episodes of fever and sore throat. Complete blood count (CBC) showed abnormal low of the count of red blood cell, hematocrit, hemoglobin, and lymphocytes. With recurrent attacks of these symptoms in a regular manner and serial $C B C$, the patients were diagnosed with cyclic neutropenia. Figure 1 shows the neutrophil level of the two patients among different times.

The symptoms were fluctuating almost every 3 weeks and continued for 1 week. During the episode period, they had difficulty in eating and keeping good oral hygiene. When neutropenic episodes were severe, they were admitted to the hospital, and appropriate treatment such as intravenous (IV) fluids with antipyretic, antibiotics, and G-CSF was given to them. 


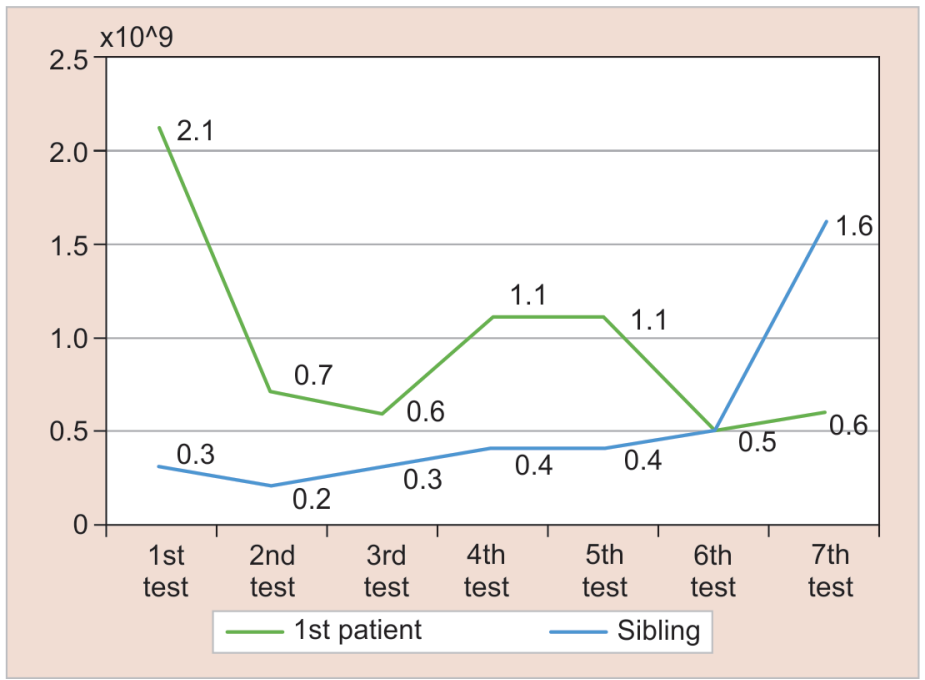

Fig. 1: Absolute neutrophils count of both patients in different occasions
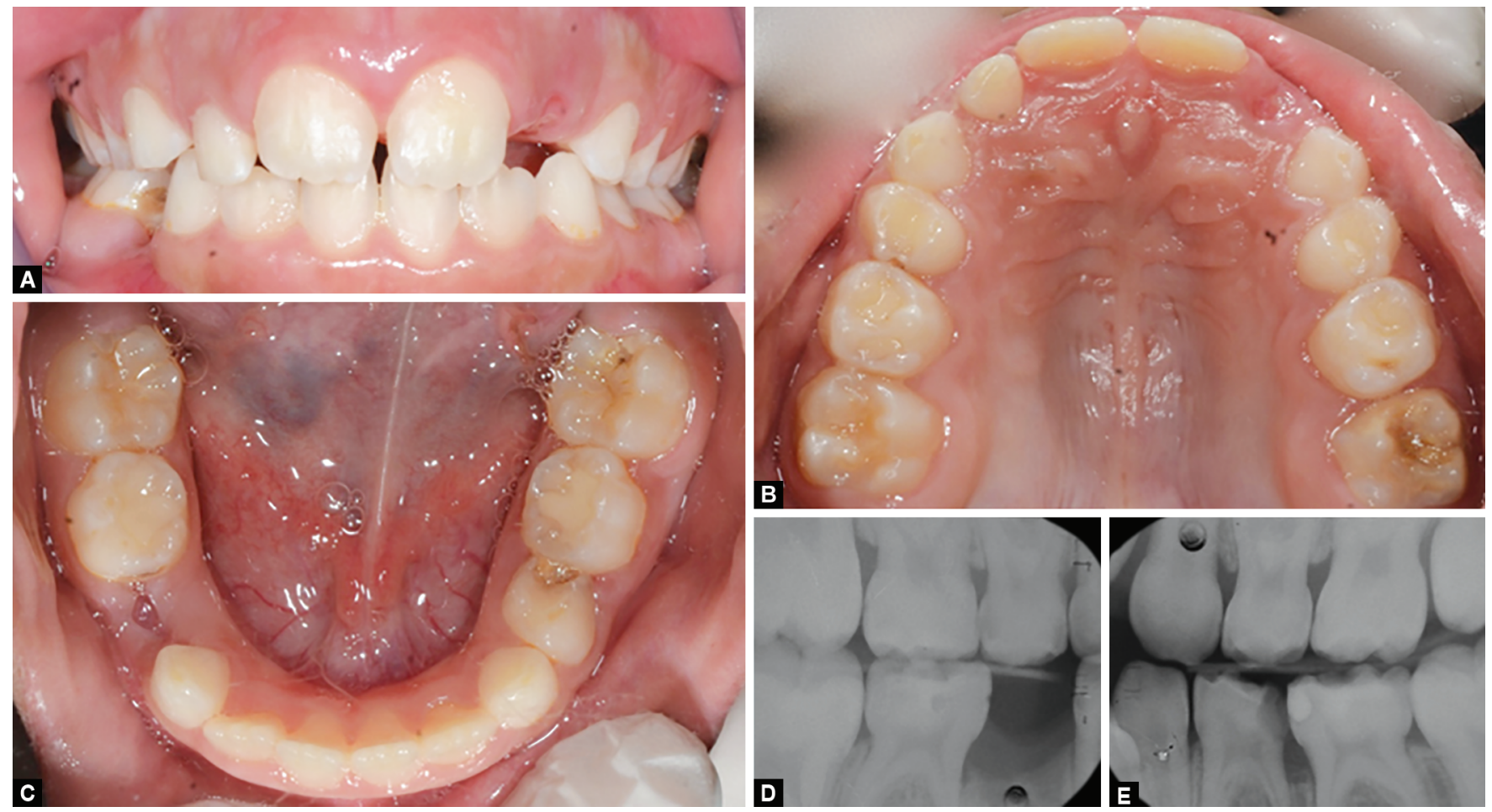

Figs 2A to E: Intraoral examination of the first patient. (A) Frontal photograph; (B) Maxillary arch occlusal photograph shows missing of \#62, and enamel hypoplasia of \#26; (C) Mandibular arch occlusal photograph shows occlusal restoration of \#75, deep carious lesion of \#74, and missing of \#84; (D) Right bitewing radiograph shows proximal caries of \#54 and 85; (E) Left bitewing shows old restoration and recurrent caries of \#75, occlusodistal carious lesion and interradicular radiolucency of tooth \#74

The dental history obtained indicated that both patients had dental visits before. For the first patient, preventive dental treatment, glass ionomer restoration, and remaining root extraction were done, whereas for the twin, only preventive dental treatment was done.

In their first dental visit extraoral and intraoral examination was carried out for both patients. Intraoral photographs for the first patient (Figs $2 \mathrm{~A}$ to $\mathrm{C}$ ) were taken in addition to two bitewings and one periapical radiographs (Figs $2 \mathrm{D}$ and $\mathrm{E}$ ). For the twin, intraoral photographs (Figs $3 \mathrm{~A}$ to $\mathrm{C}$ ) and two bitewings radiographs also were taken (Figs 3D and E). These photographs and radiographs show the important oral findings for both patients. In this visit, there was no mouth ulceration since ANC was $1.4 \times 10^{9}$ and $1.01 \times 10^{9}$ according to the blood test they did in the same day.

A preventive dental program was planned to be done using basic behavior guidance techniques. Because of the need for full dental treatment, their uncooperative behavior in the clinic and instability neutrophils level, it was decided to complete the surgical and restorative treatment in one visit under general anesthesia (GA). For preparation of dental treatment, patients were admitted in the 


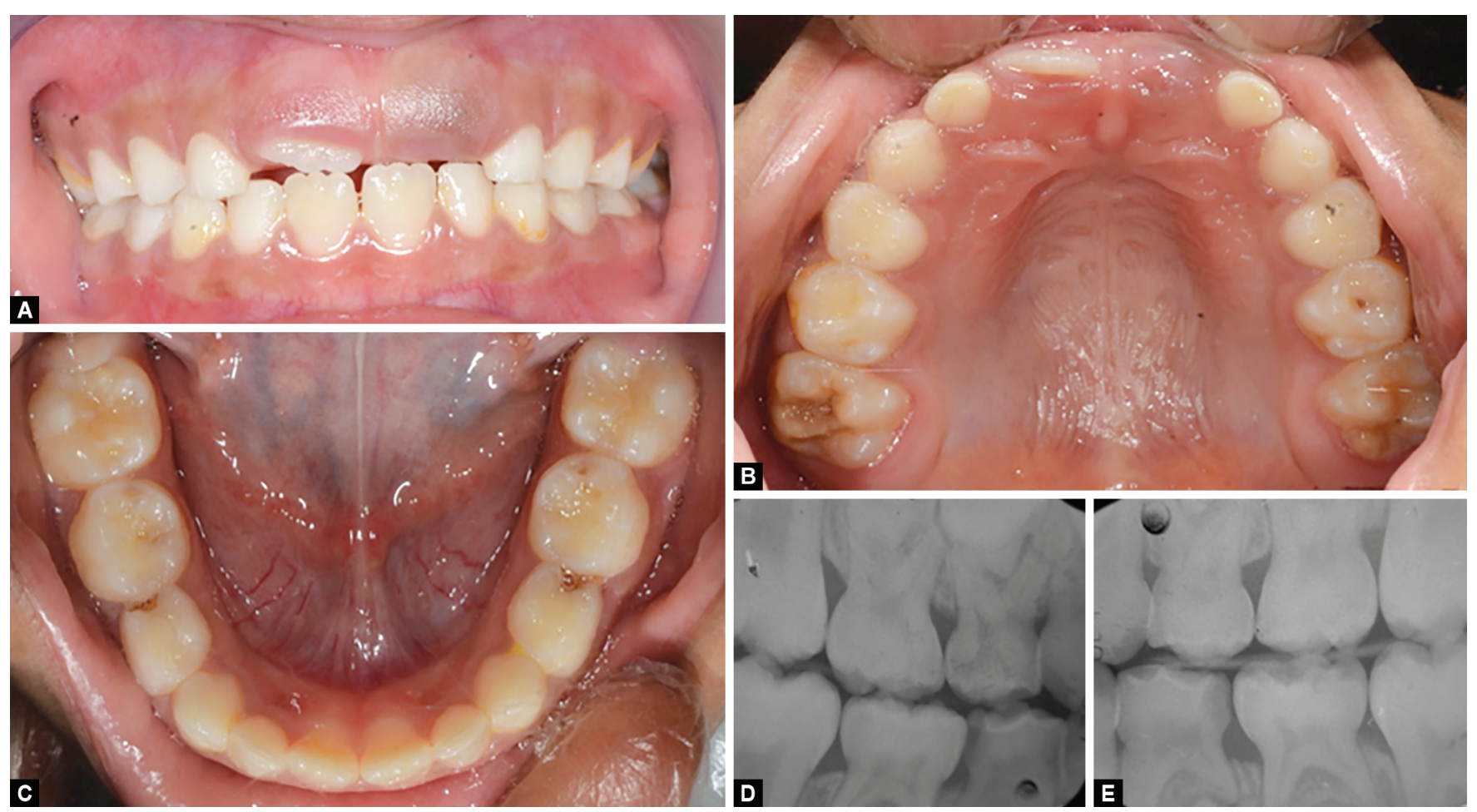

Figs 3A to E: Intraoral examination of the twin patient. (A) Frontal photograph shows erupting upper central incisors; (B) Maxillary arch occlusal photograph shows enamel hypoplasia of \#16 and 26, and missing of \#62; (C) Mandibular arch occlusal photograph shows deep occlusodistal cavity of \#74 and 84, (D) Right bitewing radiograph shows proximal carious lesions of \#54 and 84; (E) Left bitewing shows deep proximal caries of \#74

Table 1: Summary of dental treatment of both patients under general anesthesia

\begin{tabular}{|c|c|c|c|c|c|c|c|}
\hline \multicolumn{4}{|c|}{ First patient } & \multicolumn{4}{|c|}{ The twin } \\
\hline Tooth no.* & Treatment & Tooth no. & Treatment & Tooth no. & Treatment & Tooth no. & Treatment \\
\hline 16 & FS & 55 & FS & 16 & RMGl restoration & 55 & FS \\
\hline 54 & SSC & 64 & SSC & 54 & FS & 64 & FS \\
\hline 65 & $\begin{array}{l}\text { Composite } \\
\text { restoration }\end{array}$ & 26 & RMGI restoration & 65 & $\begin{array}{l}\text { Composite restora- } \\
\text { tion }\end{array}$ & 26 & RMGI restoration \\
\hline 36 & $\begin{array}{l}\text { Composite } \\
\text { restoration }\end{array}$ & 75 & $\begin{array}{l}\text { Pulpotomy** and } \\
\text { SSC }\end{array}$ & 36 & FS & 75 & $\begin{array}{l}\text { Composite } \\
\text { restoration }\end{array}$ \\
\hline 74 & Extraction & 83 & $\begin{array}{l}\text { Composite } \\
\text { restoration }\end{array}$ & 74 & Pulpotomy and SSC & 83 & $\begin{array}{l}\text { Composite } \\
\text { restoration }\end{array}$ \\
\hline 85 & $\begin{array}{l}\text { Composite } \\
\text { restoration }\end{array}$ & 46 & FS & 85 & Pulpotomy and SSC & 46 & FS \\
\hline
\end{tabular}

*Teeth numbering using ISO system; **Pulpotomy done using MTA; RMGI, resin-modified glass ionomer

hospital 2 days before the surgery. The CBC showed that ANC for the first patient was 1,760 cells $/ \mathrm{mm}$ and for the twin was 960 cells $/ \mathrm{mm}$. According to their hematologist recommendation, $30 \mathrm{mg} / \mathrm{kg} / \mathrm{day}$ of augmentin ( $500 \mathrm{mg}$ of amoxicillin sodium and $200 \mathrm{mg}$ potassium clavulanate) and $5 \mathrm{mg} / \mathrm{kg} /$ day subcutaneous G-CSF for 2 days before the dental treatment were started. In the day of surgery, patients were transferred from the main pediatric ward to the operation room calm and premedicated accompanied by their parents. General anesthesia was induced via nasal intubation, throat pack was placed, and time out was started. Table 1 shows the details of full dental treatment completed under GA. The treatment included restorative treatment, pulp therapy, and simple extractions. Restorative treatment included restorations using glass ionomer cement (GIC), composite resin restorations, stainless steel crowns (SSCs), and fissure sealant (FS).
As pulp therapy, mineral trioxide aggregate (MTA) pulpotomy for teeth with reversible pulpitis was done. Finally, nonrestorable teeth were extracted in a traumatic way.

After completion of dental treatment, mouth was cleaned, throat pack removed, patient extubated, and transferred to the recovery room. Subsequently, they were transferred to the main pediatric ward, another CBC was taken, and ANC was 1,800 cells/ $\mathrm{mm}$ for the first patient and 960 cells/mm for the twin. When both patients were fully alert, awake without pain, vomiting, or bleeding, they were discharged in healthy and stable conditions. Paracetamol $125 \mathrm{mg} / 5 \mathrm{~mL}$ syrup ( $5 \mathrm{~mL}$ when necessary needed) and augmentin $250 \mathrm{mg} / 5 \mathrm{~mL}$ syrup ( $10 \mathrm{~mL}$ every 12 hours) were prescribed for them as home medication. The two patients were then discharged after they met discharge criteria. 
One week later, both patients came for posttreatment follow-up. On examination, the restorations were intact without detection of any caries, and soft tissue was healthy. For the first patient, lower lingual arch (LLA) as a space maintainer was indicated because of the bilateral space loss in the lower arch. So, fabrication of the appliance was started and then delivered 1 week later. After 3 months of treatment, both patients came for follow-up. In this visit, the first patient complained of discomfort and multiple ulcerations related to the bands of LLA (Fig. 4). The LLA was removed and reinforcement of oral hygiene instructions

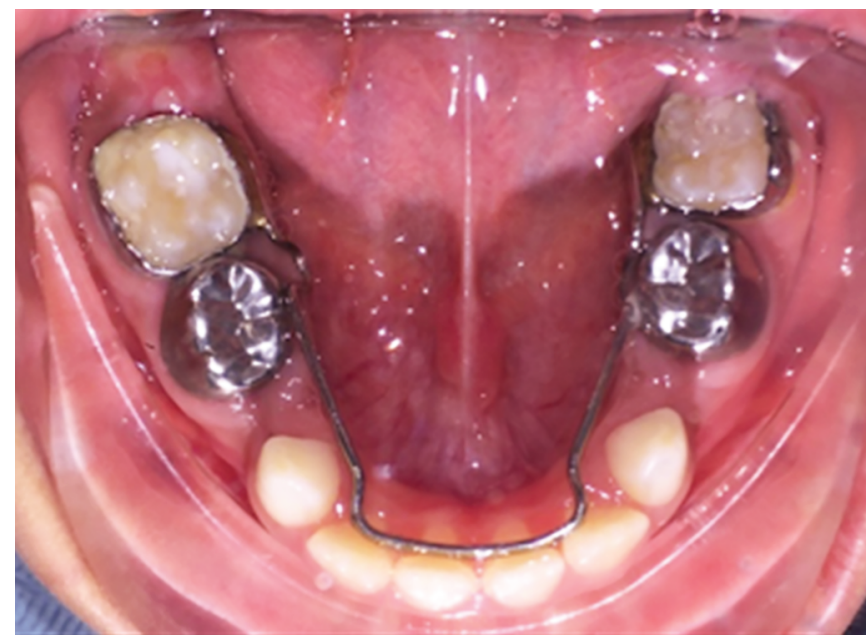

Fig. 4: Mandibular arch occlusal view of first patient shows mouth ulceration related to the bands of lower lingual arch space maintainer
$(\mathrm{OHI})$, and prescription of $0.12 \%$ chlorhexidine gluconate was done. Later on, the LLA appliance was replaced with bilateral band and loop as a less traumatic space maintainer.

Afterward, patients with their parents came for 6 months recall. In this visit, attrition of the GIC restoration on tooth 26 for the first patient was noticed, and so SSC for the same tooth was done. In the six months recall visit, dental treatment results were assessed clinically (Figs $5 A$ to $C$ ) and radiographically (Figs 5D and E) for the first patient. Also, for the twin, intraoral photographs (Figs $6 \mathrm{~A}$ to $\mathrm{C}$ ) and radiographs (Figs $6 \mathrm{D}$ and $\mathrm{E}$ ) were taken in the sex months recall visit to assess dental treatment. These photographs and radiographs reveal intact restorations and healthy soft and hard oral tissues.

\section{Discussion}

This report describes dental treatment of fraternal twin girls diagnosed with cyclic neutropenia. The diagnosis was made based on their medical history, clinical evaluation, and serial measurements of neutrophils in multiple occasions. Cyclic neutropenia is autosomal-dominant disorder, and diagnosis can be confirmed genetically by testing mutations in the ELANE gene. ${ }^{6}$ Cyclic neutropenia has a wide spectrum severity. According to clinical manifestations and the multiple blood tests for current cases, the severity of their condition is moderate to severe. Oral manifestations noticed in these cases were generalized mild gingivitis, recurrent aphthous ulcers, and oropharyngitis. These manifestations were prescribed in previous cases, ${ }^{5,7,8}$ but the manifestations here are milder than other cases. This can be justified by the differences in severity and the regular follow-up of
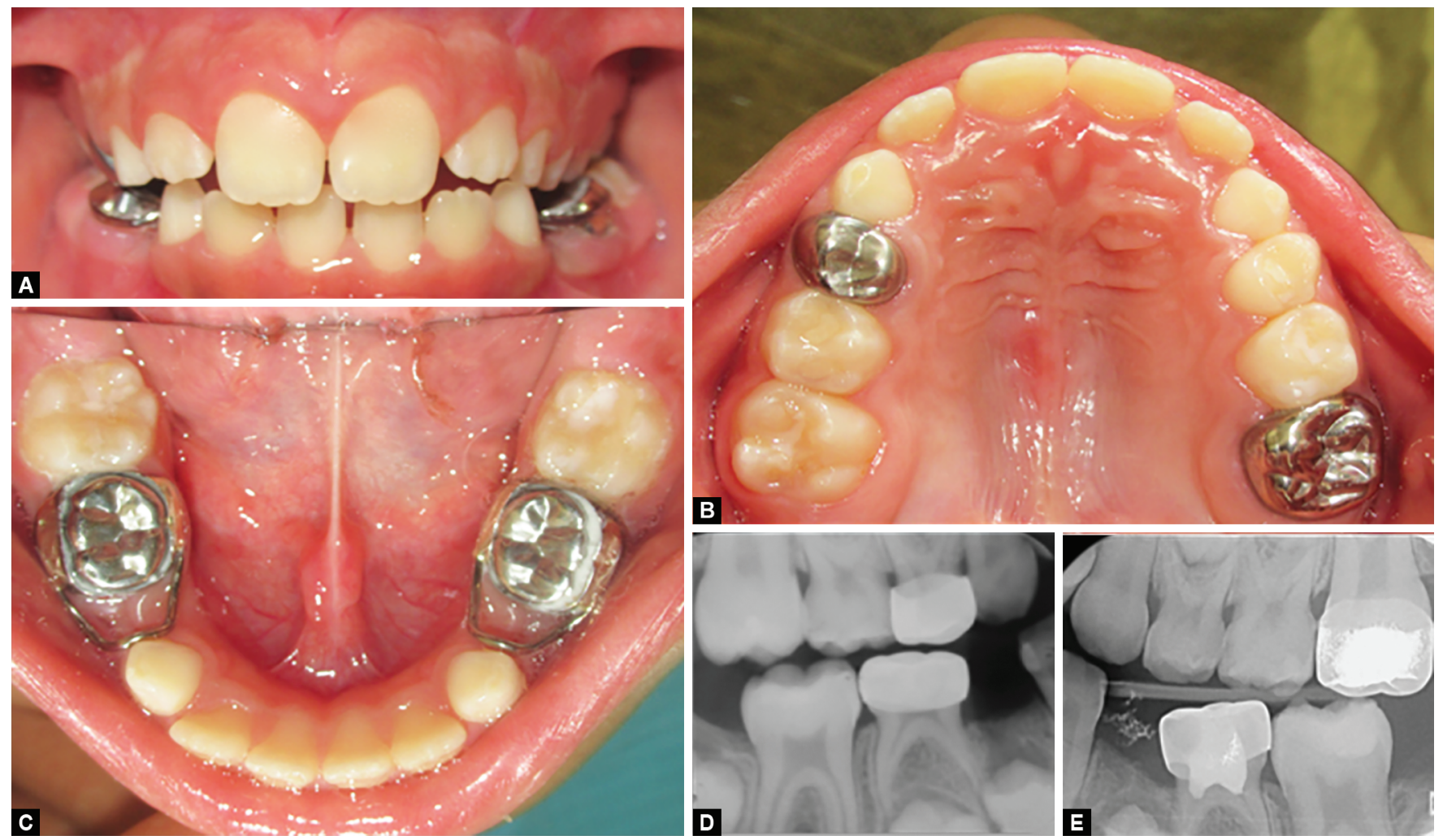

Figs 5A to E: Intraoral examination of the first patient in the six months recall visit. (A to C) Frontal, maxillary arch occlusal, and mandibular arch occlusal photographs respectively show healthy soft and hard tissues and intact restorations; (D) Right bitewing radiograph shows intact SSC of \#54 and 85; (E) Left bitewing radiograph shows SSC of \#26 and pulpotomy and SSC of \#75 

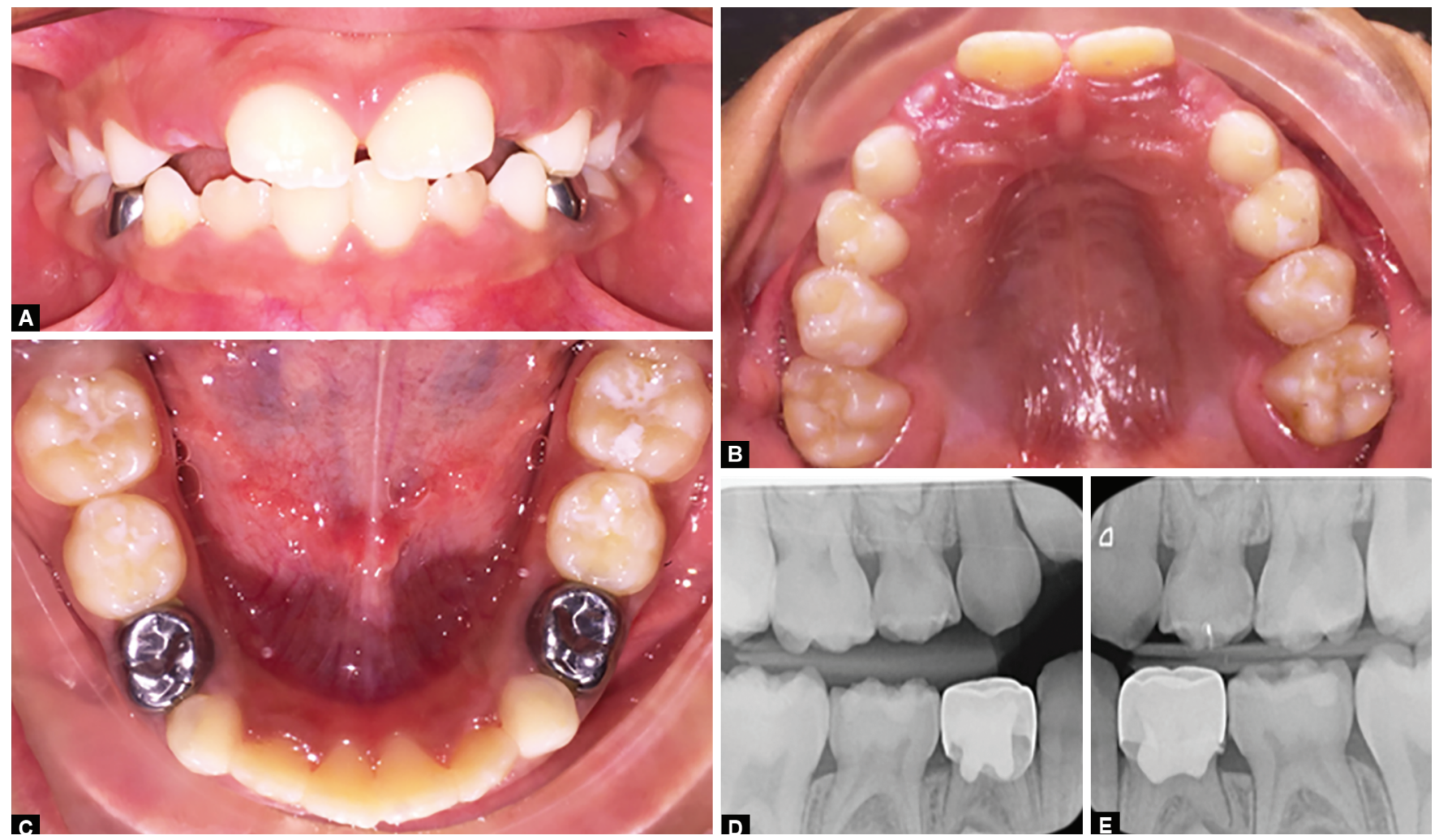

Figs $6 \mathrm{~A}$ to E: Intraoral examination of the twin patient in the six months recall visit. (A to C) Frontal, maxillary occlusal, and mandibular occlusal photographs respectively show healthy soft and hard tissues and intact restorations; (D) Right bitewing radiograph shows pulpotomy and SSC of \#84; (E) Left bitewing radiograph shows pulpotomy and SSC of \#74

the patients. Parents' awareness of the problem plays an important role in preventing more serious manifestations and complications.

Management of oral manifestations in the current cases included supportive treatment of recurrent ulcers and nonsurgical periodontal treatment. This includes professional removal of plaque, periodontal maintenance every 3 months, and daily rinsing with $0.12 \%$ chlorhexidine gluconate during the neutropenic episodes. Such minimum invasive periodontal treatment is essential to prevent periodontal infections and decrease the need for surgical intervention in children. ${ }^{9}$ Surgical periodontal treatment in severe cases with cyclic neutropenia has been reported previously. ${ }^{10}$

In the present cases, instead of treating only oral manifestations of the disease, full mouth treatment of the patients was completed. After completion of the treatment, elimination of infection, reduction of pain, and restoration of occlusion and function were achieved. Both patients came afterward in regular dental visits to check dental treatment results and to help the patients in reducing bacterial plaque accumulation. It has been reported that mainlining adequate oral hygiene in addition to periodontal treatment may result in improvement of systemic health of neutropenic patient. ${ }^{11}$ Even for patients who received medical management for neutropenia, decreased bacterial plaque by periodontal therapy may result in restoration of normal ANC level. ${ }^{12}$

Because of the medical condition of the patients, dental treatment was completed under GA. Patients with complex medical conditions who needs invasive dental procedures are candidates for treatment under GA. ${ }^{13}$ It was recommended by the hematologist to give both patients subcutaneous G-CSF for 2 days before the operation. Treatment by G-CSF was described to increase the median of ANC and to reduce cyclic neutropenia severity and duration. ${ }^{14}$ Before dental treatment, IV augmentin was given to both patients as a prophylactic antibiotic. Prescribing prophylactic antibiotic for immunocompromised patient is recommended according to American Academy of Pediatric Dentistry. ${ }^{15}$ Posttreatment additional doses of antibiotic were prescribed also because of their compromised host response. These cases were followed up for 6 months for prevention and establishment of good oral hygiene.

\section{Conclusion}

This report emphasizes the importance of dental treatment for patients with rare medical conditions. The oral cavity is an important source of inflammation or infection, which could have a major effect on systemic health. In patients with cyclic neutropenia, the oral cavity often mirrors systemic health since mouth ulceration and gingival inflammation is main manifestation. Nonsurgical periodontal treatment with regular periodontal maintenance is effective to maintain good oral health of patients with cyclic neutropenia.

\section{References}

1. Hoffbrand V, Mossn P. Hoffbrand's Essential Haematology, 7th ed., Oxford: Blackwell Science; 2015.

2. Dale DC, Bolyard AA, Aprikyan A. Cyclic neutropenia. Semin Hematol 2002;39(2):89-94. DOI: 10.1053/shem.2002.31917. 
3. Lange RD, Jones JB. Cyclic neutropenia. Review of clinical manifestations and management. Am J Pediatr Hematol Oncol 1981;3(4):363-367.

4. Carlsson G, Wahlin YB, Johansson A, et al. Periodontal disease in patients from the original Kostmann family with severe congenital neutropenia. J Periodontol 2006;77(4):744-751. DOI: 10.1902/ jop.2006.050191.

5. Chen $Y$, Fang $L$, Yang $X$. Cyclic neutropenia presenting as recurrent oral ulcers and periodontitis. J Clin Pediatr Dent 2013;37(3):307-308. DOI: 10.17796/jcpd.37.3.n8k0111177074828.

6. Horwitz MS, Corey SJ, Grimes HL, et al. ELANE mutations in cyclic and severe congenital neutropenia: genetics and pathophysiology. Hematol Oncol Clin North Am 2013;27(1):19-41. DOI: 10.1016/ j.hoc.2012.10.004.

7. Nakai Y, Ishihara C, Ogata S, et al. Oral manifestations of cyclic neutropenia in a Japanese child: case report with a 5-year follow-up. Pediatr Dent 2003;25(4):383-388.

8. Lu RF, Meng HX. Severe periodontitis in a patient with cyclic neutropenia: a case report of long-term follow-up. Chin J Dent Res 2012;15(2):159-163.

9. Hakki SS, Aprikyan AA, Yildirim S, et al. Periodontal status in two siblings with severe congenital neutropenia: diagnosis and mutational analysis of the cases. J Periodontol 2005;76(5):837-844. DOI: 10.1902/jop.2005.76.5.837.
10. Rylander $\mathrm{H}$, Ericsson I. Manifestations and treatment of periodontal disease in a patient suffering from cyclic neutropenia. J Clin Periodontol 1981;8(2):77-87. DOI: 10.1111/j.1600-051X.1981. tb02347.x.

11. Tozum TF, Berker E, Ersoy F, et al. The relationship between periodontal status and peripheral levels of neutrophils in two consanguineous siblings with severe congenital neutropenia: case reports. Quintessence Int 2003;34(3):221-226.

12. Goultschin J, Attal U, Goldstein M, et al. The relationship between peripheral levels of leukocytes and neutrophils and periodontal disease status in a patient with congenital neutropenia. J Periodontol 2000;71(9):1499-1505. DOI: 10.1902/jop.2000.71. 9.1499 .

13. Chen YP, Hsieh CY, Hsu WT, et al. A 10-year trend of dental treatments under general anesthesia of children in Taipei Veterans Genera Hospital. J Chin Med Assoc 2017;80(4):262-268. DOI: 10.1016/ j.jcma.2016.11.001.

14. Hammond 4th WP, Price TH, Souza LM, et al. Treatment of cyclic neutropenia with granulocyte colony-stimulating factor. N Engl J Med 1989;320(20):1306-1311. DOI: 10.1056/ NEJM198905183202003.

15. American Academy of Pediatric Dentistry. Antibiotic prophylaxis for dental patients at risk for infection. Pediatr Dent 2014;36(special issue):287-292. 